11,16

\title{
Приближенный метод описания эволюции, предшествующей коалесценции
}

\author{
(C) В.Б. Курасов \\ Санкт-Петербургский государственный университет, \\ Санкт-Петербург, Россия \\ E-mail: victor_kurasov@yahoo.com \\ Поступила в Редакцию 16 июля 2019 г. \\ В окончательной редакции 16 июля 2019 г. \\ Принята к публикации 25 июля 2019 г.
}

\begin{abstract}
Предложен новый приближенный метод для описания эволюции системы на стадии после окончания существенного потребления метастабильной фазы и до начала коалесценции. Проанализированы свойства эволюции в течение этого периода и показано почему асимптотика Лифшица-Слезова достигается столь быстро.
\end{abstract}

Ключевые слова: фазовые переходы первого рода, кинетика, коалесценция.

DOI: $10.21883 /$ FTT.2020.01.48729.54ks

\section{1. Введение}

Знаменитая асимптотическая теория Лифшица-Слезова [1] (ЛС) описывает поведение системы в фазовом переходе первого рода на финальной стадии, когда зародыши больших размеров потребляют метастабильную фазу столь активно, что частицы малых размеров начинают растворяться. Замечательным фактом является универсальный характер первых двух членов в асимптотическом разложении. Позже Осипов и Кукушкин нашли регулярную асимптотику [2] для асимптотического поведения характеристик системы на финальной стадии эволюции.

Определенный интерес представляет вопрос, как система достигает ЛС-асимптотики. Эксперименты показывают, что это происходит достаточно быстро [3], но объяснение этому отсутствовало. Целью настоящей публикации является представление приближенной аналитической модели, которая показывает, как происходит приближение к асимптотическому решению. Эта модель является очевидным упрощением подхода, начатого в [4] и развитого в [5-8]. Но недавний пересмотр упомянутых подходов позволяет обобщить теорию и дать новый рецепт выбора переменной, которая приводит к правильному поведению критического размера. Проведенный анализ структурирован следующим образом:

- вначале мы выделим необходимые черты из теоретического описания стадии нуклеации;

- затем мы постоим форму спектра размеров на стадии диффузионного размытия;

- далее мы сравним полученную форму с формой спектра размеров в ЛС-теории и увидим приближенную похожесть;

— чтобы завершить теорию, мы проанализируем структуру решения в ЛС-теории и увидим путь вы- бора подходящей переменной в нашем приближенном решении.

\section{2. Характерные масштабы}

Мы начинаем анализ со стадии образования основного количества сверхкритических зародышей новой фазы (стадии нуклеации). Наша цель - показать, что спектр размеров новых зародышей в момент окончания существенного потребления избыточной метастабильности будет достаточно узким в переменных, которые обеспечивают независимость формы спектра размеров пока зародыши продолжают свой рост как свехкритические объекты новой фазы. Несмотря на то, что количественное описание кинетики нуклеации широко известно [9] (хотя существуют и другие подходы [10]), анализ может быть проведен в достаточно общем случае посредством относительно простых оценок.

Большим параметром на стадии нуклеации будет число молекул критического зародыша (т. е. $v_{c}-$ индекс отмечает критический зародыш). Иначе термодинамическое описание критического зародыша потеряет силу, а в этом случае работа образования критического зародыша не сможет быть определена аналитически. При этом возможно предложить достаточно очевидную аппроксимацию для интенсивности нуклеации $I$ в диапазоне, где $I$ меняется в $e$ раз

$$
I\left(\xi_{1}\right)=I\left(\xi_{2}\right) \exp \left(-\frac{\partial F}{\partial \xi}\left(\xi_{1}-\xi_{2}\right)\right) .
$$

Здесь $\partial F / \partial \xi$ является производной свободной энергии $F$ образования зародыша по пересыщению $\zeta$ (см. [9]) взятой для критического зародыша, $\xi_{1,2}$ являются двумя значениями пересыщения и учтено, что $I \sim \exp \left(-F_{c}\right)$ в ведущем члене. Учитывая, что $F \sim \ln (\xi+1) v+\ldots$ в 
ведущем члене, приходим к

$$
I\left(\xi_{1}\right)=I\left(\xi_{2}\right) \exp \left(v_{c}\left(\xi_{1}-\xi_{2}\right) /(\xi+1)\right)
$$

Тогда относительное уменьшение $d_{\xi} / \xi=$ $=\left(\xi v_{c} /(\xi+1)\right)^{-1} \approx v_{c}^{-1}$ пересыщения означает практически прекращение нуклеации. Этот диапазон соответствует также и изменению пересыщения на стадии нуклеации, поскольку, если интенсивность изменения внешних условий за этот период существенно изменится, то это означает, что уже сформированные зародыши не смогут перебороть внешнее воздействие и стадия нуклеации не сможет закончиться. Итак, количество вещества в зародышах новой фазы к моменту окончания нуклеации может быть оценено как $q_{n}=\xi v_{c}^{-1}$. При окончании потребления избыточной метастабильности, когда пересыщение $\xi$ падает практически до нуля и критический размер начинает существенно расти, количество вещества в зародышах новой фазы может быть оценено как $q_{s}=\xi$. Чтобы увидеть, что это означает для координаты спектра размеров, мы должны посмотреть какова будет переменная, обеспечивающая независимость скорости роста зародыша от его размера. Несмотря на то, что в [11] рассмотрены много различных режимов роста зародышей, следует признать, что в большинстве ситуаций именно переменная $y=v_{c}^{1 / \alpha}$ с $\alpha \in[3 / 2,3]$ и является такой переменной. Все другие ситуации достаточно редки. Мы будем называть упомянутый интервал существенным интервалом (СИ).

Если обозначить посредством $d_{y}$ ширину спектра и посредством $f_{m}$ его амплитуду, то можем оценить $q_{n}$ как $f_{m} d_{y}^{\alpha+1}$. Величина $q_{s}$, поскольку $q_{s} / q_{n} \sim v_{c} \gg 1$, может быть оценена как $y_{s}^{\alpha} N$, где $y_{s}$ является координатой спектра размеров при окончании существенного потребления избыточной метастабильности и $N=f_{m} d_{y}$ является числом капель. Тогда приходим к $y_{s} / d_{y}=v_{c}^{1 / \alpha} \gg 1$, что означает то, что спектр размеров является относительно узким к концу потребления избыточного вещества. Основной необходимый результат и заключается в том, что спектр размеров зародышей является узким в конце стадии потребления избыточного вещества.

\section{3. Модель}

Относительно скоро (в сравнении с характерными временами коалесценции) координата узкого спектра размеров достигает окрестности критического размера. Чтобы увидеть релаксацию, достаточно решить дифференциальное уравнение первого порядка или же предпринять асимптотический анализ, что полностью описано в упомянутых публикациях. Тем не менее, результат очевиден - узкий спектр размеров останавливается при координате, равной размеру критического зародыша. Регулярная скорость здесь нулевая и можно увидеть расплывание узкого спектра размеров в силу диффузии.

На основе решения диффузионного уравнения можно увидеть трансформацию спектра размеров. Мы знаем форму решения с узким пиком в начальный момент. Это функция Грина уравнения диффузии, которая является гауссианом

$$
G(v, t) \sim \exp \left(-\left(v-v_{c}\right)^{2} /(4 D t)\right) .
$$

Здесь $D$ является коэффициентом диффузии, который в шкале выглядит как $W^{\text {inside }}+W^{\text {outside }}$ (сумма прямого и обратного потоков). Итак, мы получаем решение.

Спектр похож на гауссиан и согласно условию баланса $v_{c}$ близко к головке гауссиана. Тогда понятно, что граница области капель, которые растворяются уже достаточно быстро, будет рано или поздно достигнута существенной частью спектра.

Вначале существенная часть спектра находится в области $\left|v-v_{v}\right| \ll 1$. Тогда нет разницы, писать уравнение диффузии в -шкале или в $S=v^{2 / 3}$-шкале, или в $\rho=v^{1 / 2}$-шкале. Время, за которое существенная часть спектра достигает $v_{l}$ (размер, который растворяется достаточно быстро регулярным образом), может быть просто вычислено из $4 D t=\left(v_{c}-v_{l}\right)^{2}$. Здесь время отсчитывается от конца предыдущего периода.

Позднее условие $f\left(v_{l}\right)=0$ для спектра размеров капель $f$ становится важным. Приближенно можно взять $v_{l}^{1 / 3} \approx 0.5 v_{c}^{1 / 3}$. Функциональная зависимость $W^{\text {inside }} \sim v^{2 / 3}$ (мы рассматриваем здесь свободномолекулярный режим обмена веществом, когда нет нужды заботиться о влиянии ближайших соседей) делает предпочтительным использование $S=v^{2 / 3}$ как переменной для написания и решения уравнения диффузии.

Решение уравнения диффузии с упомянутым граничным условием понятно, это

$$
\begin{gathered}
f(S)=0 \text { для } \quad S<S_{l} \quad \text { и } \\
f(S)=G_{\text {positive }}+G_{\text {negative }} \quad \text { для } \quad S>S_{l} .
\end{gathered}
$$

Здесь $\quad G_{\text {positive }}=\frac{N}{\sqrt{4 \pi D t}} \exp \left(\frac{-\left(S-S_{c}\right)^{2}}{4 D t}\right), \quad G_{\text {negative }}=$ $=\frac{-N}{\sqrt{4 \pi D t}} \exp \left(\frac{-\left(S+S_{0}\right)^{2}}{4 D t}\right) \quad$ и $\quad S_{0}$ определено условием $S_{c}-S_{l}=S_{l}-S_{0}$ в начальный момент.

Можно увидеть, что в противоположность неограниченному решению с полушириной $d_{s} \sim t^{1 / 2}$, где амплитудная (и средняя) величина стоит на месте $S_{\max }=$ const, новое решение имеет движущуюся амплитудную координату $S_{\max } \sim t^{1 / 2}$, движущуюся среднюю величину $S_{\text {mean }} \sim t^{1 / 2}$ и растущую полуширину $d_{S} \leq t^{1 / 2}$, что обеспечивает относительную локализацию спектра. Величина пересыщения определяется из

$$
\int_{0}^{\infty} 3 \rho^{2} f(\rho)\left(\frac{d \rho}{d t}\right) d \rho=0 .
$$

Тогда получаем $\rho_{c}$ и пересыщение. Ясно, что согласно предыдущей формуле критический размер $S_{c}$ должен быть в существенном спектре. Спектр размеров хорошо локализован в шкале размеров и не может убежать 


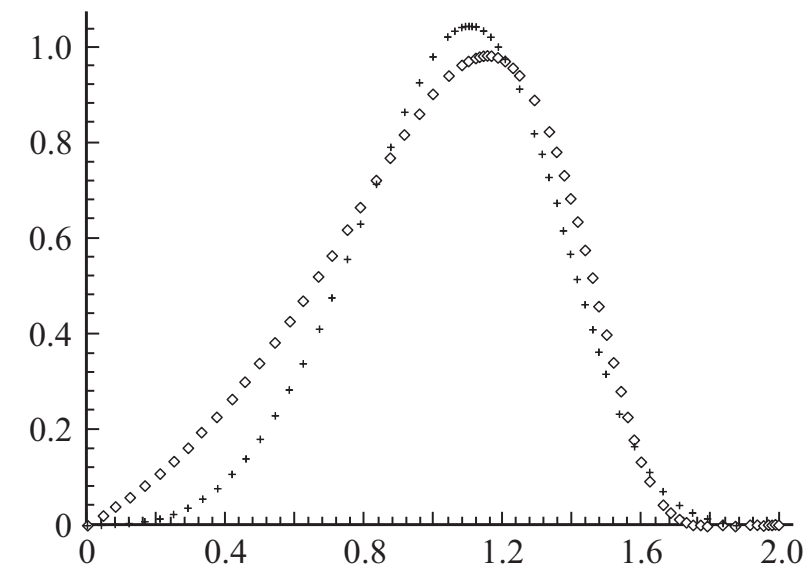

Рис. 1. Двойное гауссовское распределение (ромбики) и распределение Лифшица-Слезова (крестики) в $v^{1 / 3} / v_{c}^{1 / 3}$ шкале.

от критического размера. Мы будем называть новый спектр размеров Двойным гауссовским (ДГ) спектром размеров.

\section{4. Схожесть с ЛС-формой}

Удивительным фактом является то, что ДГ-распределение очень похоже на ЛС-распределение. Это видно из рис. 1, где показаны ДГ- и ЛС-распределения в случае свободномолекулярного режима. В диффузионном режиме картина похожая.

Передний край спектра размеров определяется с некоторой ошибкой в силу дискретности числа молекул в зародыше. В любом случае область малых не важна в балансе вещества, она не может быть измерена в эксперименте с хорошей точностью, поскольку зародыши в этой области имеют существенную скорость растворения (не относительного растворения, а физического). Итак, можно заключить, что в рамках точности современного эксперимента невозможно различить ДГи ЛС-спектры. Необходимо отметить, что мы не нормировали полуширины, а только разделили распределения на их амплитудные значения.

Близость ЛС- и ДГ-спектров объясняет то, почему в экспериментах не видят долгой релаксации к ЛС-асимптотике. Эта близость важна для обоснования ЛС-асимптотики, поскольку решать аналитически эволюционное уравнение с диффузионными членами не умеем, а без диффузионных членов возникают проблемы. А теперь видим, что диффузия форму спектра размеров существенно не поменяет.

\section{5. Выбор переменных}

В ДГ-решении всё хорошо за исключением главной черты - функциональная зависимость критического размера не соответствует той, что дается ЛС-асимптотикой. Но мы уже отмечали, что спектр размеров остается умеренно узким и сконцентрирован вблизи критического размера, где регулярная скорость отсутствует и уравнение диффузии можно записать хоть в $\rho$-шкале, хоть в $v$-шкале, хоть в $S$-шкале, хоть в любой переменной $v^{\beta}$ с умеренной степенью $\beta$. Это свойство относительной независимости ДГ-модели.

Теперь дадим рецепт выбора степени надлежащим образом. Структура ЛС-асимптотического решения имеет одну приятную особенность, которая до сих пор еще не выявлялась явно, но была причиной установления правильной степени в степенной зависимости критического размера еще со времен Тодеса [12]. А именно, степень в степенной зависимости критического размера может быть получена требованием того, что сверхкритические зародыши должны иметь ту же степень во временной зависимости, что и критический размер (асимптотическое балансное свойство). Иначе критический размер превзойдет размер капель и сделает его пренебрежимым или же капли сделают критический размер относительно пренебрежимым, что также бессмысленно. Это замечание аналогично ЛС-анализу, позволяющему найти конкретное значение параметра $\gamma$ (см. [1]), но гораздо более грубое. Мы будем называть это свойство возможностью грубого анализа. Решение ЛС распадается на два шага - установление временной зависимости критического размера и определение спектра размеров (свойство декомпозиции). Первый шаг может быть выполнен в грубом приближении.

В свободномолекулярном режиме линейный размер $\rho=v^{1 / 3}$ сверхкритических зародышей растет вне зависимости от размера и пропорционально пересыщению. Тогда, выбирая $\xi \sim t^{\alpha}$ с некоторым $\alpha$, получаем $\rho \sim t^{\alpha+1}$. Для критического размера $\rho_{c} \sim \xi^{-1}$ имеем $\rho_{c} \sim t^{-\alpha}$. Итак, эквивалентность зависимостей дает $\alpha=1 / 2$. Поскольку в ДГ-решении размер выбранной переменной растет как $t^{1 / 2}$, ясно, что переменная, обеспечивающая правильную зависимость, это $y=\rho$. Именно эту переменную следует выбрать в качестве $S$ в представленном выше решении.

В диффузионном режиме обмена веществом $v^{2 / 3}=S$ растет без зависимости скорости от размера и пропорционально пересыщению $\xi$. Выбирая $\xi \sim t^{\alpha}$, получаем $S \sim t^{\alpha+1}$. Для критического размера приходим к $v_{c}^{1 / 3} \sim \xi^{-1} \sim t^{\alpha}$. Баланс асимптотик ведет к $\alpha=-1 / 3$. Соответствие между $y \sim t^{1 / 2}$ и ростом критического размера означает, что нужно выбрать $y=v^{1 / 2}$ как переменную в ДГ-решении. ДГ-модель с упомянутым рецептом выбора переменной оказывается полезным инструментом в описании эволюции системы на стадии, предшествующей коалесценции.

\section{6. Обсуждение результатов и выводы}

Как мы видели, ДГ-решение в различных режимах отличается только выбором переменных. Мы упоминали, что ДГ в определенном смысле нечувствительно к 


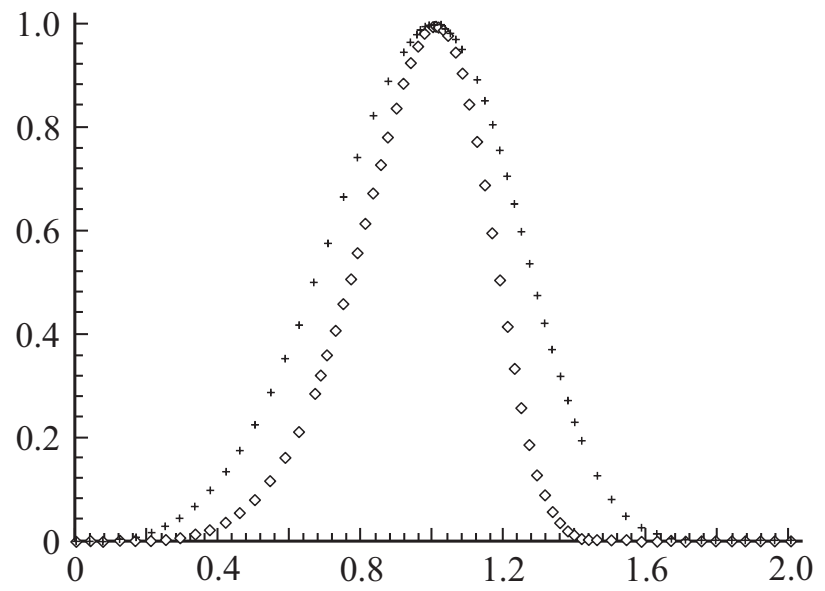

Рис. 2. ДГ-распределение для диффузионного случая (ромбики) и для свободномолекулярного случая (крестики) как функции отмасштабированного $v^{1 / 3} / v_{c}^{1 / 3}$ аргумента.

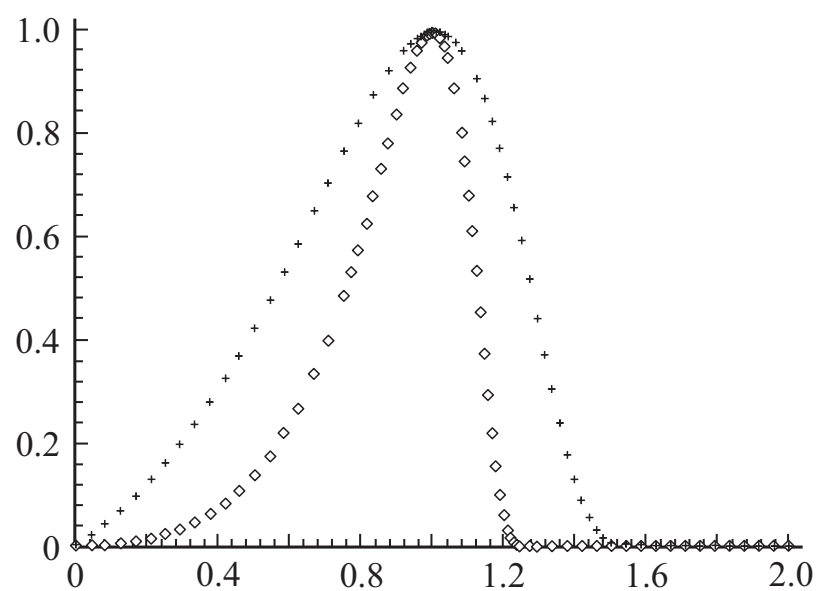

Рис. 3. ЛС-распределение для диффузионного случая (ромбики) и для свободномолекулярного случая (крестики) как функции отмасштабированного $v^{1 / 3} / v_{c}^{1 / 3}$ аргумента.

выбору переменных. Тогда следует ожидать, что разница в форме спектров размеров будет мала. Покажем спектры размеров на границах СИ и ожидаем, что спектры размеров во всех ситуациях из СИ лежат внутри граничных ситуаций. Итак, получаем рис. 2, где ДГ-распределения нарисованы для свободномолекулярного и диффузионного режимов, и видим приближенную похожесть. Уже упомянутая близость ЛС- и ДГ-спектров размеров позволяет ожидать что также и ЛС-спектры из всего СИ будут похожи. Это видно из рис. 3, где нарисованы ЛС-спектры для свободномолекулярного и диффузионного режимов.

Итак, можно говорить о приближенной похожести ЛС-спектров размеров в СИ и о приближенной похожести ДГ-спектров в СИ. Эта похожесть подтверждает подход [13], основанный на методе наискорейшего спуска в построении спектров размеров на стадии коалесценции.

\section{Конфликт интересов}

Автор заявляет, что у него нет конфликта интересов.

\section{Список литературы}

[1] И.М. Лифшиц, В.В. Слезов. ЖЭТФ 35, 479 (1958).

[2] С.А. Кукушкин, А.В. Осипов. ЖЭТФ 113, 2193 (1998).

[3] V.V. Slezov. Kinetics of First-order Phase Transitions. WILEYVCH Verlag GmbH and Co. KgaA, Weinheim. (2009). 432 p.

[4] V.B. Kurasov. Development of the universality conception in the first order phase transitions. Chemistry Research Institute of Sankt-Petersburg State University, St. Petersburg. (1998). $125 \mathrm{p}$.

[5] V. Kurasov. General trends of the late period of evolution in the quasichemical model of nucleation. www.arXiv.org arXiv:cond-mat/0607768. $16 \mathrm{p}$.

[6] V. Kurasov. Different scenarios of the late stages of condensation. arXiv:0801.0239v1 [cond-mat.stat-mech].

[7] V. Kurasov. Late periods of the condensation process. arXiv:1011.3543v1 [cond-mat.stat-mech].

[8] V.B. Kurasov. Advances in the first order phase transitions. V. Agglomeration, Thermal effects, Multiple channels. Overcondensation, Kinetic peculiarities of nucleation. Art-Xpress Publishing, Saint-Petersburg. (2016). 322 p.

[9] В.Б. Курасов. Теорет. мат. физика 131, 503 (2002).

[10] S. Kukushkin, A. Osipov. J. Chem. Phys. 107, 3247 (1997).

[11] С.А. Кукушкин, В.В. Слезов. Дисперсные системы на поверхностях твердых тел: механизмы образования тонких пленок. Наука, Санкт-Петербург. (1996). 304 с.

[12] О.М. Тодес. В кн: Проблемы кинетики и катализа. Изд-во AH CCCP, M. (1949). C. 137-173.

[13] V.B. Kurasov. Universality in kinetics of the first order phase transitions. Chemistry Research Institute of Sankt-Petersburg State University, St. Petersburg (1997). 400 p.

Редактор Д.В. Жуманов 